\title{
Análisis de la Identidad Laboral y el sin Sentido en Relación al Trabajo Tercerizado
}

\section{Bruno Sánchez-Flores}

Estudiante de la Maestría en Psicología del Trabajo y las Organizaciones, Becario CONACYT

\section{Crishelen Kurezyn-Díaz}

Candidata a Doctora en Dirección de Organizaciones

\section{Manuel González-Pérez}

$\mathrm{PhD}$. Ingeniería. Sistema Nacional de Investigadores (SNI-1) Universidad Popular Autónoma del Estado de Puebla (UPAEP), México

\section{doi: 10.19044/esj.2017.v13n8p85 URL:http://dx.doi.org/10.19044/esj.2017.v13n8p85}

\begin{abstract}
Labor identity (LI) is a fundamental aspect of the worker. Without it, we are prone to generate nonsense for our work. Labor Outsourcing (LO) is a form of work that encourages the loss of LI and it has as a consequence on the nonsense of work (NW). The aim of this research is to investigate the correlation between LI and loss of consciousness in the LO context. To address this research, a quantitative research was conducted. A total sample of 55 people, composed of men and women between 25 and 56 years old, was used. Respondents were working on the outsourcing scheme in the personnel supply modality. The instrument that was applied was based on individual elaboration. Findings on the basis of NW and LI show high positive rates. Based on this, we can say that, despite the fact that workers live this precarious modality of work, they are motivated. However, we found that this absence of NW and presumed LI as a function of LO, is actually an apparent factor. This is due to the key items I2, SS3, T1 and T5 that has helped us to find the necessary support to affirm the existence of an aspirational LI feeling that generates the meaning of the work. Finally, we conclude that LO is perceived by the sample as a possible means to be directly employed by the primary organization of work.
\end{abstract}

Keywords: Labor Outsourcing, Labor Identity, Nonsense of work

\section{Resumen}

La identidad laboral (IL) es un aspecto fundamental del trabajador. Sin esto, somos proclives a generar un sin sentido (SS) por nuestra labor. La 
Tercerización laboral (TL) es una forma de trabajo que fomenta la pérdida de IL y tiene como consecuencia un SS del trabajo. El objetivo de esta investigación es indagar sobre la correlación que existe entre la IL y pérdida de sentido en el marco de la TL. Para abordar esta investigación, se realizó una investigación cuantitativa. Se usó una muestra total de 55 personas, compuesta por hombres y mujeres entre 25 y 56 años. Los participantes encuestados se encontraban trabajando en el esquema de TL en la modalidad de suministro de personal. El instrumento que se aplicó es de elaboración propia. Los hallazgos en función del SS y la IL muestran altos índices positivos al respecto. Con base en esto, podemos decir que, a pesar de que los trabajadores viven esta modalidad precaria de trabajo, se muestran motivados. Sin embargo, encontramos que esta ausencia de SS y presunta IL en función de la TL, en realidad es un factor aparente. Debido a los ítems clave I2, SS3, T1 y T5 que nos ayudaron a encontrar el sustento necesario para afirmar la existencia de un sentimiento aspiracional de IL que genera el sentido de la labor. Finalmente concluimos en que la TL es percibida por la muestra como un posible medio para poder ser contratado de manera directa por la organización primaria de trabajo.

Keywords: Tercerización Laboral, Identidad Laboral, Sin Sentido

\section{Introduction}

\section{Identidad laboral}

El sentido de identidad es una parte fundamental en los individuos. Es un aspecto que determina los rasgos de nuestra percepción dentro de la sociedad. Para Giménez (2010), la identidad del individuo es un proceso subjetivo y auto reflexivo en función de los atributos culturales. Por otro lado, Salszman (2013), menciona que, además la identidad individual supone un proceso de identificación e internalización de roles y estatus.

Como parte de esta cultura e internalización de roles, es importante tener en cuenta el rol que se tiene en la dimensión del trabajo. Es necesario considerar este constructo de ideas para poder establecer un concepto de identidad laboral. Padilla (2016), menciona que nuestra identidad está constituida en función de las diferentes facetas de nuestra vida. De tal forma que, nuestra identidad se construye con base en dimensiones como la política-ideológica, filosófica, cultural, profesional y laboral.

De esta forma, podemos ver que la identidad es un conglomerado de facetas que convergen en un punto. Creando así, sentido pertenencia, categorización y representación social (Delgadillo, Vargas, Nievar, Arguello y González, 2013; citados por García, Carreón, Hernández, Aguilar y Rosas, 2015) 
Es así que, al hablar de un contexto laboral inestable, se propicia la pérdida de IL. El ejemplo de inestabilidad laboral está representado en la TL. Aunque, el modelo de TL por sí mismo no supone un impacto en la IL.

La inserción y reinserción precaria de las personas en las empresas coadyuva a la pérdida de IL. Aunado a esto, Juliao, Sánchez y Martínez (2014) mencionan que los rendimientos marginales decrecientes y la desmotivación, sean las consecuencias naturales al no existir un contrato psicológico. Es decir, un nexo de pertenencia con una organización. El cual, se extingue en el modelo de TL. Pero es necesario definir las formas de trabajo tercerizado que existen.

\section{Tercerización laboral}

El neoliberalismo ha sentado las condiciones para que se geste la TL. En un primer acercamiento, se le considera como flexibilidad en la contratación, como un insumo más que "propicia” que las organizaciones cuenten con menores restricciones para incorporar a un creciente número de personas en una actividad económica subordinada (Arregui, 2016: p. 175).

Aunado a esto, Márquez y Revelo (2013), mencionan que, la globalización económica ha tenido un impacto significativo en la transformación del mercado laboral. Este cambio se ha efectuado a través de la inserción en la economía, promoviendo nuevos empleos para la sociedad e incluyendo nuevas formas de contratación laboral, obteniendo como resultado la TL.

Pero es necesario establecer concretamenmte la definición de TL. Álvarez (2012) define la tercerización del empleo como una técnica de gestión; en la cual, se subcontratan empresas externas las cuales fungen como proveedoras para llevar a cabo una actividad.

Este modelo de TL puede ser adoptado desde dos modalidades. Es decir, la subcontratación laboral y el suministro de trabajadores por la vía de una empresa de trabajo temporal (Ugarte, 2006).

Este régimen de TL supone también una forma de precariedad laboral. A este respecto, Sotelo (1999) sostiene que la TL es una forma de precarización del empleo. Ya que, los trabajadores son despojados de sus derechos y diluidos en células sin poder de negociación.

Por lo tanto, se puede encontrar una correlación entre la pérdida de IL que estaría asociada a la forma de TL. Ya que, el flujo constante de personal estaría asociado a una pérdida de identidad. Es así que, la TL supone inestabilidad e inserción precaria en el trabajo. Estos elementos crean una barrea tácita para el trabajador subcontratado. Por lo tanto, la crisis de IL repercute en la pérdida de sentido del trabajador. 


\section{Sin Sentido}

El SS es una consecuencia de la crisis de IL. Neal y Groat, citados por Robinson, Shaver y Wrightsman (2013) ven al SS como un elemento de la alienación. Es decir, la falta de sentido que percibe un individuo en relación con un tema social.

El SS puede impactar en la calidad de trabajo del individuo. Ya que, el SS está relacionado con el nivel de compromiso en el trabajo. Esto no necesariamente se refiere a un grado de desinterés o irresponsabilidad. Pero es más probable que, una persona con bajo nivel de compromiso aporte lo mínimo requerido. Siguiendo con esta línea, Puentes \& Gómez (2012), plantean que el compromiso del trabajador estará ligado a la identificación y reconocimiento de sus acciones, orientadas a garantizar algún tipo de servicio. De esta manera, existirá la disposición de abarcar más allá de las propias responsabilidades, aportando algún servicio o producto de forma satisfactoria y proporcionando un sentido.

Con base en Romero (2016), la metamorfosis del trabajo permite que el significado y sentido sean percibidos de manera diferente. Existen algunas variables como calidad de vida laboral, bajas expectativas de promoción y desarrollo en el lugar de trabajo que impactan en la configuración del sentido del trabajo. De esta manera, la TL que implica ciertas condiciones de trabajo, ya sean flexibles o precarias inciden en la IL. Es así que, La TL en la modalidad de suministro de personal, y la crisis de IL generan una actividad alienante y carente de sentido.

\section{Metodología}

Este estudio usa el enfoque cuantitativo. Se usó una muestra total de 55 personas. Esta muestra está compuesta por hombres y mujeres entre 25 y 56 años. Los participantes encuestados se encontraban trabajando en el esquema de TL mediante la modalidad de suministro de personal.

El diseño del instrumento aplicado es de elaboración propia. Se construyó con base en las categorías de identidad laboral (IL), sinsentido (SS) y tercerización laboral (TL). El instrumento se compone de 15 ítems agrupados de la siguiente manera:

\begin{tabular}{|c|c|}
\hline Dimensión & Reactivos \\
\hline \multirow{4}{*}{ Identidad Laboral } & $\begin{array}{l}\text { Me siento parte de la empresa donde } \\
\text { trabajo a pesar de estar subcontratado. }\end{array}$ \\
\hline & $\begin{array}{cc}2 . & \text { Las personas subcontratadas son } \\
\text { tratadas de manera diferente en comparación con } \\
\text { quienes sí pertenecen a la empresa. }\end{array}$ \\
\hline & $\begin{array}{l}\text { 3. Estas diferencias en el trato impactan } \\
\text { de forma negativa el desempeño de mi labor. }\end{array}$ \\
\hline & $\begin{array}{cc}4 . \quad \text { Me relaciono mejor con mis } \\
\text { compañeros subcontratados que con los } \\
\text { compañeros que trabajan directamente para la }\end{array}$ \\
\hline
\end{tabular}




\begin{tabular}{|c|c|}
\hline & empresa. \\
\hline & $\begin{array}{l}\text { 5. Tengo acceso a las dinámicas extra } \\
\text { labores de la empresa donde trabajo. } \\
\end{array}$ \\
\hline \multirow{6}{*}{ Sin Sentido } & $\begin{array}{l}\text { 1. } \\
\text { Al no pertenecer a la empresa directa } \\
\text { siento que mi labor no significa nada. }\end{array}$ \\
\hline & $\begin{array}{cc}2 . & \text { Las funciones que desempeño } \\
\text { actualmente están acordes con mis habilidades y } \\
\text { aspiraciones laborales. }\end{array}$ \\
\hline & $\begin{array}{l}\text { 3. Si fuera contratado/a directamente por } \\
\text { la empresa donde me desempeño (contrato } \\
\text { estable) me sentiría más comprometido con mi } \\
\text { labor. } \\
\end{array}$ \\
\hline & $\begin{array}{l}\text { Además del aspecto económico, trabajo } \\
\text { por la satisfacción que representa mi labor. }\end{array}$ \\
\hline & $\begin{array}{l}\text { 5. Trabajo por los incentivos económicos } \\
\text { que puedo alcanzar a través de mi labor. }\end{array}$ \\
\hline & $\begin{array}{l}\text { 6. La labor que realizo me genera un } \\
\text { sentimiento de crecimiento personal y } \\
\text { profesional. }\end{array}$ \\
\hline \multirow{5}{*}{ Tercerización Laboral } & $\begin{array}{l}\text { 1. Prefiero trabajar subcontratado y la } \\
\text { flexibilidad de compromiso que representa. }\end{array}$ \\
\hline & $\begin{array}{l}\text { 2. Estoy satisfecho/a con las prestaciones } \\
\text { que tengo como trabajador subcontratado. } \\
\end{array}$ \\
\hline & $\begin{array}{l}\text { 3. Como trabajador subcontratado cuento } \\
\text { con seguros / garantías (médicos o legales) que } \\
\text { me protejan y me den seguridad para desempeñar } \\
\text { mi labor. }\end{array}$ \\
\hline & $\begin{array}{c}4 . \quad \text { Trabajar bajo el esquema de } \\
\text { subcontratación impacta negativamente en mi } \\
\text { nivel personal, familiar y económico. }\end{array}$ \\
\hline & $\begin{array}{c}\text { 5. Prefiero un trabajo estable y las } \\
\text { prestaciones que ello implica (generar } \\
\text { antigüedad, caja de ahorro, protección sindical, } \\
\text { posibilidad de pensión, etc.) }\end{array}$ \\
\hline
\end{tabular}

\section{Fuente: Elaboración Propia}

Para las respuestas, se optó por una escala Likert con cinco opciones. 5 es totalmente de acuerdo, 4 de acuerdo, 3 ni de acuerdo ni en desacuerdo, 2 en desacuerdo y 1 totalmente en desacuerdo.

Para la validación del instrumento se realizó un pilotaje aplicado a 20 participantes. Hombres y mujeres de entre 21 y 45 años. Los participantes encuestados se encontraban trabajando en el esquema de TL mediante la modalidad de suministro de personal.

La confiabilidad del instrumento de medición se llevó a cabo mediante el uso del modelo matemático Anova. El análisis de los datos se realizó a través del software Microsoft Excel en su versión 2016. 


\begin{tabular}{|c|c|c|c|c|c|c|}
\hline \multicolumn{7}{|c|}{$\begin{array}{l}\text { ANÁLISIS DE } \\
\text { VARIANZA }\end{array}$} \\
\hline $\begin{array}{c}\text { Origen de las } \\
\text { variaciones }\end{array}$ & $\begin{array}{c}\text { Suma de } \\
\text { cuadrados }\end{array}$ & $\begin{array}{c}\text { Grados de } \\
\text { libertad }\end{array}$ & $\begin{array}{l}\text { Promedio de } \\
\text { los cuadrados }\end{array}$ & $F$ & $\begin{array}{l}\text { Proba } \\
\text { bilidad }\end{array}$ & $\begin{array}{l}\text { Valor } \\
\text { crítico } \\
\text { para F }\end{array}$ \\
\hline Entre grupos & 63.95727 & 54 & 1.18439389 & $\begin{array}{c}0.627 \\
11566\end{array}$ & $\begin{array}{c}0.9835 \\
2661\end{array}$ & $\begin{array}{c}1.3535612 \\
2\end{array}$ \\
\hline $\begin{array}{l}\text { Dentro de los } \\
\text { grupos }\end{array}$ & 1452.3619 & 769 & 1.88863707 & & & \\
\hline Total & $\begin{array}{c}1516.3191 \\
7\end{array}$ & 823 & & & & \\
\hline
\end{tabular}

\section{Fuente: Elaboración propia}

Como el valor calculado está dentro del valor crítico significa que la hipótesis nula se acepta con un P (0.05) y por lo tanto, las medias de los encuestados son iguales.

Se efectuó una segunda medición de confiabilidad mediante la técnica Alfa de Cronbach. Se ocupó el Software estadístico SPSS en su versión 22.

\begin{tabular}{|c|c|c|}
\hline \multicolumn{3}{|c|}{ Estadísticas de fiabilidad } \\
\hline Alfa de Cronbach & $\begin{array}{c}\text { Alfa de Cronbach basada en } \\
\text { elementos estandarizados }\end{array}$ & N de elementos \\
\hline .674 & .704 & 21 \\
\hline
\end{tabular}

\section{Fuente: Elaboración propia}

Oviedo y Campos (2005) rescatados de Machorro, Rosado y Romero (2011) mencionan que los valores de Alfa superiores a 0.7 son suficientes para garantizar la fiabilidad de la escala.

\section{Resultados y Discusión}

\section{Media General por Categorías}

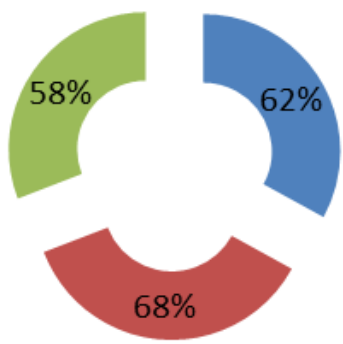

- Identidad

(3.13454545454545)

- Sin Sentido

(3.46181818181818)

- Tercerización

(2.97804713804714) 


\section{Fuente: Elaboración Propia}

La dimensión de identidad tiene 62\%. Equivale a una media de 3.13. Esto muestra una tendencia al alta en el nivel de identidad de la muestra. Por otro lado, encontramos que existe un mayor nivel de sentido en relación con la dimensión de SS. Finalmente, la percepción de la muestra en relaciona a la TL presenta un decremento.

A primera vista, podría parecer que la percepción de la TL en la muestra no está minando su identidad y sentido laboral.

A pesar de que los datos muestran una tendencia a la alta general en función de la identidad. Estos son los hallazgos al analizar las dimensiones por separado.

\section{Análisis por Nivel de Identidad}

nivel bajo (Menor a 2.5) Nivel medio (Entre 2.6 y 3.5 )

Nivel alto (Entre 3.6 y 5 )

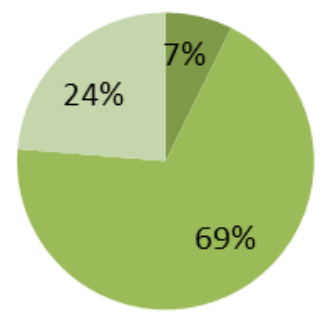

\section{Fuente: Elaboración Propia}

El nivel de identidad tiende con un 69\% hacia el nivel medio de acuerdo. Mientras que el $24 \%$ de la muestra se encuentra con un alto nivel de identidad. Finalmente, el $7 \%$ considera no sentirse identificado con la empresa.

En primera instancia se asume que el nivel de identidad del 69\% de la muestra tiene una alta tendencia hacia el reconocimiento con la empresa. Sin embargo, es necesario analizar los resultados por ítem para constatar esta afirmación. 


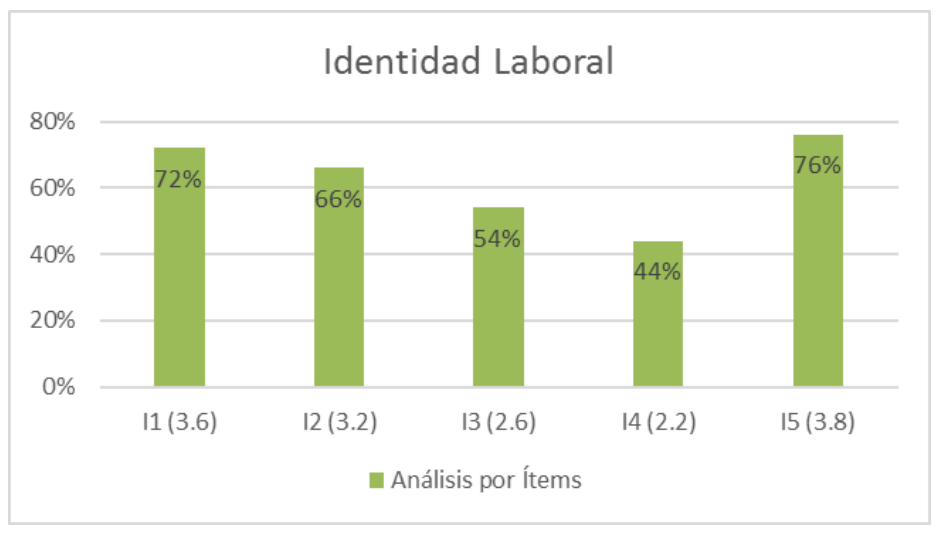

\section{Fuente: Elaboración Propia}

I1 se refiere al sentido de identificación de la muestra con la empresa. Los datos nos dicen que existe un $72 \%$ de reconocimiento con la empresa a pesar de estar subcontratados

I2 se refiere a la percepción del trato diferencial que reciben los subcontratados. Podemos ver que, existe un 66\% de acuerdo con esta afirmación.

I3 pregunta si existe un impacto negativo en relación con el trato diferencial. Con un 54\% de acuerdo podemos decir que el impacto negativo no repercute dramáticamente en la percepción de la muestra.

I4 busca encontrar si existe una diferencia en la relación entre las personas subcontratadas y las que pertenecen a nómina. Con un $44 \%$ de acuerdo, podemos ver que no existe mucha diferencia entre los pares en la organización.

I5 busca encontrar los niveles de identificación con la empresa que subcontrata. Podemos decir que existe un alto grado de identificación con los valores de la empresa que subcontrata.

De acuerdo a esta información podríamos inferir que, las personas subcontratadas tienen un nivel alto de identidad. Pero el análisis del segundo ítem nos dice que estas personas son conscientes de que existe un trato diferencial.

A pesar de sentirse identificados con la empresa detectamos un factor divisorio. Este factor que encontramos es vital para demostrar que el sentido de pertenencia que nos arrojan los números de la media general de identidad puede ser en apariencia.

El factor puede estar en función de la barrera tácita que existe en relación a la cultura organizacional. La cual, les impide permearse de la misma. Por lo tanto, hablaríamos de un deseo de pertenencia, es decir una identidad aspiracional. 
Es así que, las personas subcontratadas de la muestra se reconocen como ajenos. A pesar de que afirmen sentirse identificados con la empresa. Por lo tanto, y en función de la perspectiva teórica de identidad en la que nos basamos, no existe un sentido de IL definida.

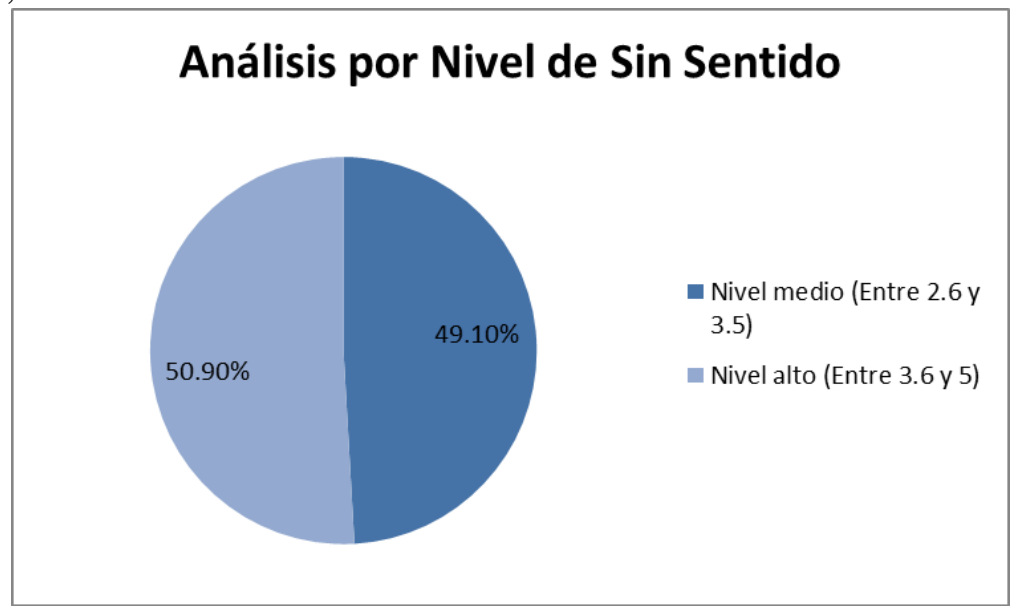

\section{Fuente: Elaboración Propia}

Se puede apreciar que la muestra tiende al acuerdo en función del sentido. El 49.1\% de los encuestados se encuentra en un nivel medio con tendencia a la alta en el acuerdo. Mientras que, el 50.9\% dice tener un alto nivel de sentido por su labor.

A continuación se presentan los resultados por ítem que compone esta dimensión.

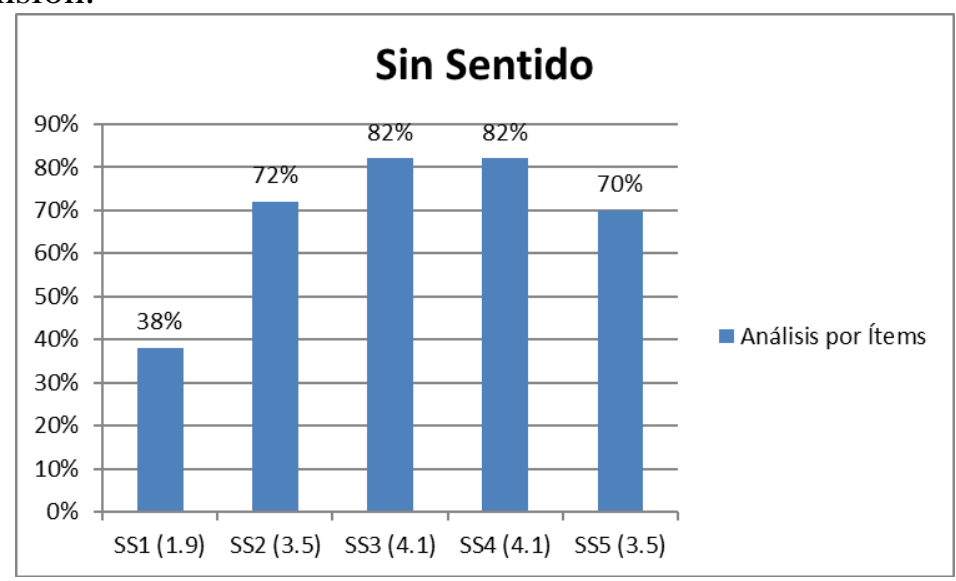

\section{Fuente: elaboración propia}

SS1 explora la relación entre el sin sentido y la subcontratación. Con un 38\% de acuerdo, vemos que la población niega tener un impacto negativo en su labor por ser subcontratada. 
SS2 indaga el hecho de que el SS se diera por que las habilidades y aspiraciones personales no estén en alineadas. El 72\% de la muestra afirma que sus habilidades y aspiraciones si están en sintonía con su puesto.

SS3 pregunta sobre el nivel de compromiso personal en relación con un contrato estable. El 82\% de la muestra afirma que su nivel de compromiso sería mayor si obtuviera un contrato directo.

SS4 se refiere al nivel de esfuerzo invertido más allá de las exigencias requeridos por el puesto. De igual manera, el $82 \%$ de la muestra dice esforzarse más allá de lo requerido.

SS5 pregunta si las personas trabajan por los incentivos económicos que pueden alcanzar. Con un ligero decremento en el nivel de acuerdo, el $70 \%$ de la muestra dice trabajar por los incentivos económicos.

$\mathrm{Al}$ revisar estos datos, podemos decir que la población analizada tiene un alto sentido para con su labor. Es necesario preguntar $\dot{i} A$ que puede deberse esta tendencia alta de sentido en la labor subcontratada?

Para responder a esta pregunta, es importante fijarse en los ítems SS3 y SS4. Los cuales nos dicen que las personas están basando su sentido y se esfuerzan más allá, por este deseo de pertenencia.

Aquí podemos ver una conexión que vincula la identidad aspiracional mencionada anteriormente, con este alto sentido de labor detectado en la muestra encuestada.

Por otro lado, se percibe un decremento en cuanto al ítem SS5, que de alguna manera sustenta la idea de que las personas están más motivadas por pertenecer directamente a la empresa que por el incentivo económico dentro del régimen de subcontratación.

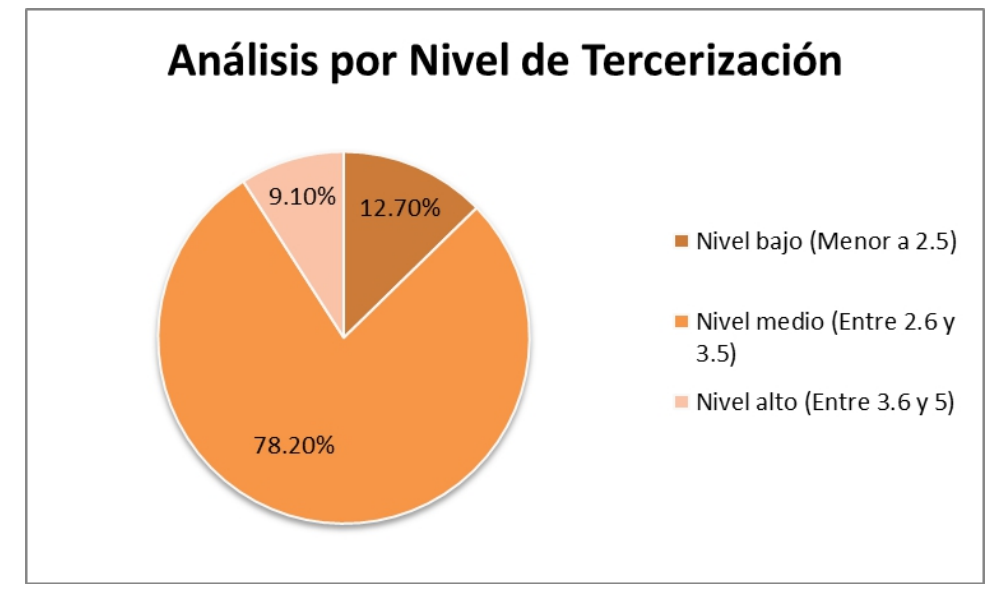

\section{Fuente: Elaboración Propia}

En esta gráfica se puede observar que el $78.2 \%$ de la muestra se encuentra en un nivel medio de acuerdo con tendencia a la alta. Por otro 
lado, el $12.7 \%$ tiene una percepción negativa sobre la TL. Mientras que el 9.1\% tiene una percepción positiva al respecto.

En el siguiente gráfico analizamos los ítems individualmente.

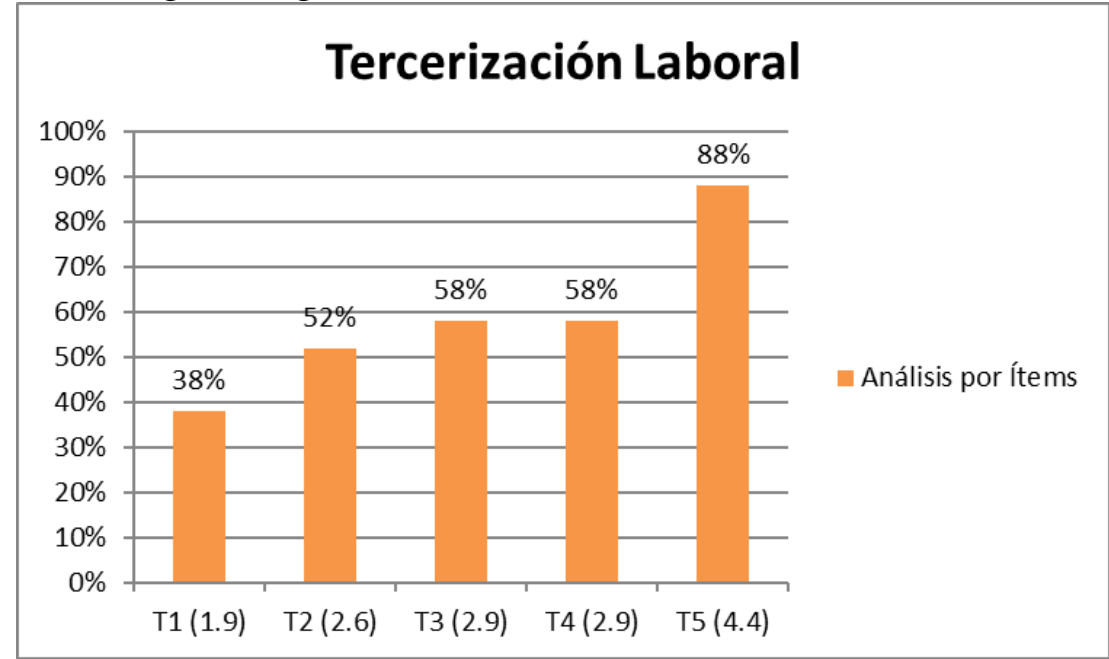

\section{Fuente: Elaboración Propia}

T1 busca establecer el nivel de acuerdo con respecto del trabajo tercerizado en función de la flexibilidad que representa. Solo el 38\% de la muestra estaría de acuerdo en trabajar mediante subcontrato.

T2 indaga el nivel de satisfacción del trabajador subcontratado en relación a las prestaciones de este modelo. El 52\% de la población dice estar conforme con las prestaciones que cuenta.

T3 pregunta por la presencia de garantías que le son brindados al trabajador en el desempeño de su labor. El 58\% de la muestra dice contar con las garantías necesarias que le protegen y le brindan seguridad para desempeñar sus funciones.

T4 establece el nivel de impacto negativo a nivel personal, familiar y económico del trabajador. El 58\% de los encuestados considera que existe una consecuencia negativa en relación con el subcontrato.

T5 pregunta sobre la disposición de conseguir un trabajo estable. Con el $88 \%$ de los encuestados a favor, se puede ver la inclinación positiva a tener un empleo estable.

Después de analizar estos datos, vemos que destaca en puntaje el ítem T5. Ya que, el $88 \%$ de la muestra se decanta por un esquema de contrato estable. Por lo tanto, podemos decir que, a pesar de que la media general sobre la tercerización laboral pareciera positiva, los datos a detalle demuestran que esto no es así. 
Esto se sustenta con los resultados obtenidos para T1. Siendo así que, el porcentaje de personas que prefieren el trabajo subcontratado es de solo $38 \%$.

Sin embargo, podemos ver que para los ítems T2 y T3, los resultados se mantienen apenas por encima del 50\%. Esto nos dice que las personas no están del todo conformes con las prestaciones con las que cuentan. De esto podemos decir que a pesar de la precariedad que puede representar, las personas estarían aceptando la subcontratación como un medio para obtener la contratación directa con la empresa.

\section{Conclusion}

El objetivo de esta investigación era corroborar la correlación negativa entre la IL el SS en relación con una forma de trabajo precario como lo es la TL en su modalidad de suministro de personal a las empresas del sector primario de trabajo.

Partimos de la idea que expresaba Sotelo sobre la precariedad laboral que representa la tercerización. Esta modalidad de contrato constituye una forma de violencia laboral. Ya que, busca despojar al trabajador de sus derechos y privilegios.

Como consecuencia de esto, conjeturamos la carencia de IL. Ya que, al no ser parte estable de una organización, las personas pueden experimentarse como extraños dentro de las mismas y ser objeto de marginación y violencia.

A este respecto, Jáuregui (2011) menciona que la violencia laboral representa un factor en detrimento de la identidad y las capacidades del trabajador. La consecuencia de esta violencia se traduce en una experiencia de aislamiento y marginación.

Este sentimiento de extrañeza y marginación, puede influir en el nivel de desempeño del trabajador. Ya que, está correlacionado con el sentido del trabajo.

Sin embargo, los hallazgos en función del SS y la IL muestran altos índices positivos al respecto. Con base en esto podemos decir que, a pesar de que los trabajadores viven esta modalidad precaria de trabajo, se muestran motivados.

Encontramos que esta ausencia de SS y presunta IL en función de la TL en realidad es un factor aparente. Debido a los ítems clave I2, SS3, T1 y T5 que nos ayudaron a encontrar el sustento necesario para afirmar la existencia de un sentimiento aspiracional de IL.

Este sentimiento aspiracional de IL puede ser la razón real del alto nivel de sentido de los trabajadores encuestados. A pesar de trabajar en una modalidad de trabajo precario, es decir la TL. 
Finalmente podemos aseverar que la TL es percibida por la muestra como un posible medio para poder ser contratado de manera directa por la organización primaria de trabajo.

Sin embargo, esta motivación podría estar fundamentada tan solo en la consecución del empleo estable. Lo que podría tener repercusiones negativas a largo plazo nuevamente en materia de SS e IL.

Por lo tanto, es necesario identificar el motor principal de la motivación. Ya que, si la motivación del individuo está en función de una meta definida, la consecución de la misma representaría la disminución paulatina de la motivación. Esto podría generar un círculo vicioso que situé al trabajador en una dinámica de estímulo y respuesta que se aleja totalmente del sentido de la labor.

En una investigación posterior, se profundizará al respecto del sentido del trabajo. Se buscará encontrar si la razón principal de que las personas se subcontraten es por la mera consecución del contrato estable, o si existe un factor diferente que les impulse más allá de este elemento externo; El sentido de su labor.

\section{References:}

1. Arregui, V. (2016). Precarización del empleo y régimen neoliberal. México. Alegatos. N. 92.

2. Caraballo, M. P. R. (2016). Significado del Trabajo y Sentido de la Profesión en la Academia en Colombia. Colombia. Athenea digital, 16(2), 427-435.

3. Esparrragoza, D. J., Bejarano, C. S., \& Díaz, D. J. M. (2014). Diferencias del clima laboral entre empleados contratados por outsourcing y empleados directos: evidencia empírica de la zona norte de Colombia. Universidad \& Empresa, 16(26), 277-295.

4. Giménez, G. (2010). Cultura, identidad y procesos de individualización. Identidades: Teorías y métodos para su análisis. México. UNAM.

5. García, J., Fuentes, J.A., Silva, A. \& Flores , M.L. (s.f.).La subcontratación laboral, su abuso y su reglamentación en México. México.

6. Jáuregui, I. P. (2011). Abuso de poder y violencia laboral. Argentina. Universidad de Flores.

7. Lirios, C. G., Guillén, J. C., Valdés, J. H., Fuentes, J. A. A., \& Ferrusca, F. J. R. (2015). Modelo de los determinantes psicosociales de la identidad laboral y la vivienda en exmigrantes de Xilitla, San Luis Potosí (Centro de México). Revista Internacional de Investigación en Ciencias Sociales, 11(1), 55-69. 
8. Márquez, L., \& Revelo, M.A. (2013). Globalización económica y transformaciones laborales: el papel de las normas internacionales de trabajo. México. Vniversitas Estudiantes. No. 10.

9. Padilla, L. (2016). Cultura e identidad nacional.México. Intuición, $1(1)$.

10. Puentes, R. C., \& Gómez, L. R. H (2012). ¿El Sentido del Trabajo o “Trabajo Sin Sentido”?/La Deseducación Colombiana: Todos Somos Culpables. Revista de Psicologia GEPU, 1(3), 7-20.

11. Ramos, F. M., Morales, M. R., \& Ortíz, M. V. R. Diseño de un instrumento para evaluar el clima organizacional en un complejo petroquímico del Estado de Veracruz. México.

12. Robinson, J. P., Shaver, P. R., \&Wrightsman, L. S. (Eds.). (2013). Measures of Personality and Social PsychologicalAttitudes: Measures of Social PsychologicalAttitudes.USA. AcademicPress, (1).

13. Salszman, M. G. (2013). Identidad, subjetividad y sentido en las sociedades complejas. Mexico. Flacso.

14. Sotelo, A. (1999). Globalización y precariedad del trabajo en México. México.El Caballito.

15. Ugarte, J. L. (2006). Sobre relaciones laborales triangulares: La subcontratación y el suministro de trabajadores. Ius et Praxis, 12(1), $11-29$. 